\title{
Surveillance and diagnosis of plague and anthrax in Tanzania and Zambia
}

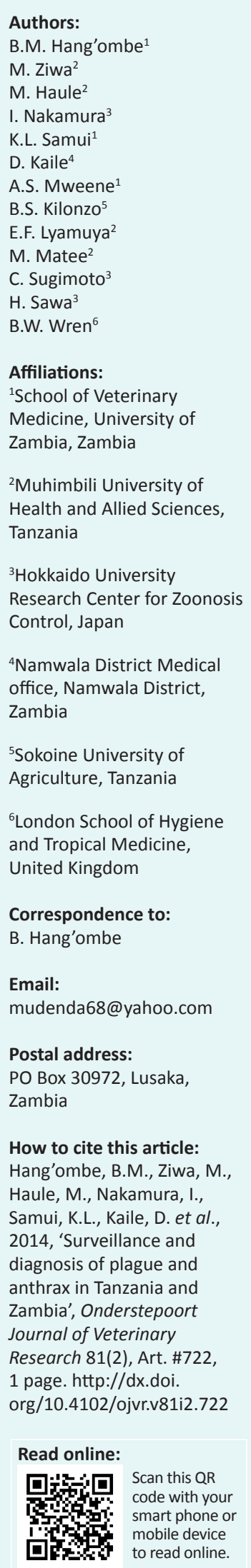

Yersinia pestis and Bacillus anthracis are diseases that rarely occur, with devastating consequences. In Africa, despite the diseases being rare, they are reported on a yearly basis in remote areas of the continent due to lack of proper surveillance and detection systems. In Tanzania and Zambia, studies have been ongoing to understand these pathogens in endemic areas.

The studies apply the well established polymerase chain reaction (PCR) for detecting Yersinia pestis DNA in suspected human specimens, rodents and their fleas by DNA extraction and primers targeting the plasminogen activator gene. In Tanzania, 516 rodent and nine human samples were analysed for the presence of Yersinia pestis DNA. Of these samples, four rodent samples belonging to Mastomys and Tatera species and two human specimens were found positive. As for Zambia, 810 rodent samples were collected and analysed. Yersinia pestis DNA was detected in 33 samples, belonging to the Tatera, Rattus and Mastomys species. As for fleas, the Xenopsylla species from rodents were positive for Yersinia pestis DNA. The isolated bacteria were subjected to antimicrobial sensitivity tests, with results indicating a response pattern as recommended by the World Health Organization in the treatment of plague. In case of anthrax, suspected samples from hippopotamuses, soil and humans were screened through bacteria culture and confirmation by PCR targeting the pXO1 (protective antigen gene) and pXO2 (capsule) virulence plasmids. Anthrax was detected in hippopotamuses, soil and humans, with the epidemiological link being confirmed through Variable Number of Tandem Repeats analysis.

The presence of Yersinia pestis DNA in rodents and fleas may represent evidence that infected rodents and fleas are being maintained in plague endemic areas, consistent with the hypothesised enzootic maintenance of plague elsewhere, whilst Bacillus anthracis is amplified by animals from soil into the human population.

\section{Acknowledgements}

This work was supported by the Wellcome Trust Grant WT087546MA to the Southern African Centre for Infectious Diseases \& Surveillance (SACIDS).

Note: Proceedings of the 2nd One Health Conference in Africa. Jointly organised by the Southern African Centre for Infectious Disease Surveillance and the Tanzania National Institute for Medical Research, held at the Snow Crest Hotel in Arusha, Tanzania from 16th to 19th April 2013: http://www.sacids.org/kms/frontend/index.php?m=119.

Copyright: @ 2014. The Authors. Licensee: AOSIS OpenJournals. This work is licensed under the Creative Commons Attribution License. 